\title{
The effect of at-birth vitamin A supplementation on differential leucocyte counts and in vitro cytokine production: an immunological study nested within a randomised trial in Guinea-Bissau
}

\author{
Mathias J. Jørgensen ${ }^{1 *}$, Ane B. Fisker ${ }^{2}$, Erliyani Sartono ${ }^{3}$, Andreas Andersen ${ }^{1,2}$, Christian Erikstrup ${ }^{4}$, \\ Ida M. Lisse ${ }^{4}$, Maria Yazdanbakhsh ${ }^{3}$, Peter Aaby ${ }^{1,2}$ and Christine S. Benn ${ }^{1,2}$ \\ ${ }^{1}$ Bandim Health Project, INDEPTH Network, Statens Serum Institut, Artillerivej 5, 2300 Copenhagen, Denmark \\ ${ }^{2}$ Bandim Health Project, INDEPTH Network, Bissau, Guinea-Bissau \\ ${ }^{3}$ Department of Parasitology, Leiden University Medical Center, Leiden, The Netherlands \\ ${ }^{4}$ Department of Clinical Immunology, Aarbus University Hospital, Skejby Sygehus, Aarbus, Denmark \\ ${ }^{5}$ Department of Pathology, Herlev University Hospital, Herlev, Denmark \\ (Submitted 7 June 2011 - Final revision received 23 February 2012 - Accepted 1 March 2012 - First published online 11 July 2012)
}

\section{Abstract}

Vitamin A supplementation (VAS) at birth was not associated with improved survival in a randomised, placebo-controlled trial in GuineaBissau. However, a negative sex-differential effect, which became evident after diphtheria-tetanus-pertussis (DTP) vaccination, was noted; among girls who had received DTP, VAS at birth was associated with two-fold higher mortality than placebo. The objective of the present study was to investigate the immunological effects of VAS at birth within a subgroup of participants in the randomised trial. Guided by the mortality results, we further explored whether VAS had a differential effect according to sex and DTP status. At 6 weeks after randomisation and supplementation, we measured differential leucocyte counts and TNF- $\alpha$, interferon- $\gamma$, IL-10, IL-13 and IL- 5 production in a whole-blood culture assay. A total of 471 children were included. VAS compared with placebo at birth was associated with a higher proportion of monocytes (relative risk ratio $1 \cdot 26,95 \%$ CI $1 \cdot 07,1 \cdot 49, P=0 \cdot 04$ ), while spontaneous TNF- $\alpha$ production was lower in the VAS group (geometric mean ratio $0.54,95 \% \mathrm{CI}, 0.37,0 \cdot 78, P=0.001$ ). Stratified analysis showed that VAS was associated with lower TNF- $\alpha$ and IL-10 production for girls without DTP and boys with DTP, resulting in significant three-way interactions between VAS, sex and DTP vaccination status ( $P=0.03$ and $P=0.04$, respectively) for spontaneous TNF- $\alpha$ and IL- 10 production. The results substantiate the potential role of VAS as an immunomodulatory intervention, which has different effects depending on concomitant health interventions and the sex of the recipient

Key words: Vitamin A supplementation: Whole-blood stimulation: Immunology: Diphtheria-tetanus-pertussis vaccination

Vitamin A is essential for immune defence and protection against infectious diseases as is seen by the severe implications of being vitamin A deficient ${ }^{(1)}$. We investigated the effect of high-dose vitamin A supplementation (VAS) with Bacille Calmette-Guérin (BCG) vaccine at birth in a randomised, double-blind, placebo-controlled trial conducted in an African setting with high infant mortality. Surprisingly, VAS was not associated with improved survival ${ }^{(2)}$. However, as seen in two preceding Asian trials ${ }^{(3,4)}$, we found a more beneficial effect in boys than in girls. The sex difference became pronounced after the children received diphtheriatetanus-pertussis (DTP) vaccinations recommended at the age of 6, 10 and 14 weeks; among girls who had received
DTP, VAS at birth was associated with $2 \cdot 19$ (95\% CI $1 \cdot 09$ 4.38) times higher mortality than placebo at birth ${ }^{(5)}$. These tendencies were subsequently confirmed in a separate study of low-birth-weight children ${ }^{(6)}$. Analysing vitamin A status evaluated by serum concentrations of retinol-binding protein (RBP) at 6 weeks as well as 4 months of age revealed no overall effect of neonatal VAS on vitamin A status, but the number of DTP vaccines received before 4 months of age was negatively associated with vitamin A status at the age of 4 months, particularly in girls ${ }^{(7)}$. A post hoc analysis revealed a strong interaction with season of supplementation, resulting in a significantly beneficial effect of VAS if given to boys during the dry season, but a significantly negative effect if

\footnotetext{
Abbreviations: atRA, all-trans-retinoic acid; BCG, Bacille Calmette-Guérin; CRP, C-reactive protein; DTP, diphtheria-tetanus-pertussis; GMR, geometric mean ratios; IFN, interferon; LLD, lower limit of detection; LPS, lipopolysaccharide; OPV, oral polio vaccine; PHA, phytohaemagglutinin; PPD, purified protein derivative of Mycobacterium tuberculosis; RBP, retinol-binding protein; Th, T helper; TT, tetanus toxoid; VAS, vitamin A supplementation.
} 
given to girls in the rainy season ${ }^{(2)}$. Hence, VAS at birth may indeed be beneficial in some circumstances. However, VAS apparently also interacts with other, yet unknown, factors including possibly sex, DTP vaccination and season, to produce a negative effect on survival. It should be essential to clarify the underlying mechanisms to optimise the use of VAS in low-income countries.

Within the randomised trial of VAS at birth, we aimed to investigate the effect of VAS at birth on the infant's developing immune system. We collected blood samples from a subgroup of the participants 6 weeks after their initial randomisation to VAS or placebo, and analysed the samples by means of differential leucocyte counts and by measuring in vitro cytokine production to various antigens and mitogens. The effect of supplementation on a potential $\mathrm{T}$ helper (Th) 1 or Th2 bias was examined by measuring the Th1 cytokine interferon (IFN) $-\gamma$ and the Th2 cytokines IL-5 and IL-13.Additionally, we examined the effects on pro-inflammatory TNF- $\alpha$ and anti-inflammatory IL-10.

We had anticipated that VAS would be associated with a reduction in mortality and wished to investigate whether any such mortality benefit would be mirrored as alterations of the cytokine profile. Based on the findings from the randomised study, we also found it relevant to explore the potential differential effect of VAS in the two sexes, and the effect of VAS according to whether DTP had been received or not. Unfortunately, the samples available for analysis were primarily collected in the rainy season (Table 1), and it was therefore not possible to examine the impact of season.

\section{Methods}

\section{Setting and population}

The Bandim Health Project has a health and demographic surveillance system in six suburban districts of the capital of Guinea-Bissau and covers approximately 90000 inhabitants. The present study was carried out within a randomised, double-blind, placebo-controlled trial of the effect of VAS with BCG vaccine at birth on mortality. Mothers giving birth at the maternity wards at the national hospital and the local health centre were invited to participate in the present study, when their child was to receive BCG vaccination after delivery. Furthermore, mothers who delivered at home were invited to participate, when they came for BCG vaccination at two of the three health centres in the study area. Inclusion criteria were weight $>2500 \mathrm{~g}$ and no signs of overt illness.

All infants were vaccinated intradermally by a trained study nurse in the upper left deltoid region with $0.05 \mathrm{ml}$ BCG vaccine (Statens Serum Institut). A total of 4345 infants were randomised to $50000 \mathrm{IU}$ vitamin A or placebo. VAS was $0.5 \mathrm{ml}$ of vegetable oil containing $50000 \mathrm{IU}$ vitamin A as retinyl palmitate and $10 \mathrm{IU}$ vitamin $\mathrm{E}$, and placebo was $0.5 \mathrm{ml}$ of vegetable oil containing only $10 \mathrm{IU}$ vitamin E. Vitamin A and

Table 1. Characteristics of the children included in the study

(Number of participants and percentages; mean values and standard deviations)

\begin{tabular}{|c|c|c|c|c|}
\hline & \multicolumn{2}{|c|}{ VAS } & \multicolumn{2}{|c|}{ Placebo } \\
\hline & $n$ & $\%$ & $n$ & $\%$ \\
\hline Blood samples obtained & 223 & & 248 & \\
\hline Differential leucocyte count performed & 221 & $0.9^{\star}$ & 246 & $0.8^{\star}$ \\
\hline Samples included in in vitro cytokine production analysis & 214 & $4 \cdot 0^{*}$ & 247 & $0.4^{*}$ \\
\hline \multicolumn{5}{|l|}{ Sex } \\
\hline Boys & 103 & $46 \cdot 2$ & 121 & $48 \cdot 8$ \\
\hline Girls & 120 & 53.8 & 127 & $51 \cdot 2$ \\
\hline \multicolumn{5}{|l|}{ Age at sampling $(d)$} \\
\hline Mean & \multirow{2}{*}{\multicolumn{2}{|c|}{$\begin{array}{c}45.2 \\
8.8\end{array}$}} & \multicolumn{2}{|c|}{$44 \cdot 9$} \\
\hline SD & & & \multicolumn{2}{|c|}{9.7} \\
\hline DTP vaccine before sampling & 57 & $25 \cdot 6$ & 48 & $19 \cdot 4$ \\
\hline OPV at birth & 156 & $70 \cdot 0$ & 178 & $71 \cdot 8$ \\
\hline Included in the dry season & 14 & $6 \cdot 3$ & 10 & 4.0 \\
\hline Included in the rainy season & 209 & 93.7 & 238 & $96 \cdot 0$ \\
\hline Positive malaria slide & 7 & $3 \cdot 1$ & 10 & 4.0 \\
\hline $\mathrm{CRP}>5 \mu \mathrm{g} / \mathrm{ml} \dagger$ & 51 & $23 \cdot 6$ & 52 & 21.5 \\
\hline Axillary temperature $>37^{\circ} \mathrm{C}$ & 10 & 4.5 & 6 & $2 \cdot 4$ \\
\hline III‡ & 59 & $27 \cdot 3$ & 61 & $24 \cdot 7$ \\
\hline Received medicine before sampling & 51 & $22 \cdot 9$ & 45 & $18 \cdot 2$ \\
\hline Paracetamol & 29 & $13 \cdot 0$ & 37 & 14.9 \\
\hline Antibiotics & 15 & $6 \cdot 7$ & 11 & 4.4 \\
\hline Chloroquin & 22 & 9.9 & 23 & $9 \cdot 3$ \\
\hline Vitamin A deficient $(<0.83 \mu \mathrm{mol}$ RBP/l) $\dagger$ & 68 & 31.5 & 79 & $32 \cdot 0$ \\
\hline Borderline vitamin A deficient $(0.83-1.11 \mu \mathrm{mol}$ RBP/I)† & 101 & $46 \cdot 8$ & 104 & $42 \cdot 1$ \\
\hline
\end{tabular}

VAS, vitamin A supplementation; DTP, diphtheria-tetanus-pertussis vaccine; OPV, oral polio vaccine; CRP, C-reactive protein; RBP, retinal-binding protein.

* Percentage lost.

†For eight children, we did not have serum available for CRP/RBP analysis.

$\ddagger$ III defined as having a positive malaria slide, elevated serum CRP, an axillary temperature above $37^{\circ} \mathrm{C}$ or any combination of these 
placebo were prepared by a Danish pharmacy, which held the code until all children had been followed for 1 year.

At the time of enrolment, information on baseline anthropometrics and socio-economic background factors was collected. The prevalence of HIV-1 among women of fertile age was $5.6 \%$ in the study area at the time of the study ${ }^{(8)}$. With the vertical-transmission control programme ongoing, we expected less than $1 \%$ of the children to be HIV-1 infected.

The Bandim Health Project provided the BCG vaccine. According to the WHO recommendations, all children should receive BCG and oral polio vaccine (OPV) at birth, followed by three DTP vaccines and OPV at 6, 10 and 14 weeks of age ${ }^{(9)}$. During the study, there were, however, periods where OPV was missing, partly because the Ministry of Health in Guinea-Bissau withheld them for two OPV campaigns conducted in October and November 2004. As a result, the children participating in the study received several different combinations of vaccines.

\section{Study design}

On 1 June 2004, we initiated the immunological subgroup study. Children included in the randomised trial were visited 6 weeks after enrolment and randomisation to VAS or placebo. The mother was informed about the subgroup study and provided consent, an interview was conducted and a blood sample obtained from the child. If a child was absent, we kept on revisiting the home until the child was found at home; however, we did not include children above 3 months of age. Initially, we attempted to only visit children who had not yet received a DTP vaccine, recommended at 6, 10 and 14 weeks of age. This left a very small window of opportunity as many children received their first dose of DTP before we were able to visit them at home. Hence, we modified the rules for inclusion; from 1 August 2004 until the end of inclusion into the trial on 28 November 2004, all children were visited regardless of whether a DTP vaccine had been registered or not.

Sample size calculations were hampered by the fact that no previous studies of the effect of neonatal VAS in this age group had been conducted. We aimed to include 200 children in each treatment group and ended up recruiting 480 children. A previous publication from our group utilised, in part, the same samples as well as samples from approximately 200 additional participants to specifically investigate the effect of VAS on the immune response to BCG vaccine ${ }^{(10)}$

At the blood sampling visits, the mother was interviewed about breast-feeding, consultations at the health centres, hospitalisations and vaccines received. Furthermore, the mother was asked about current symptoms of infections, including the use of medicine. The child's mid-upper-arm circumference and axillary temperature were measured. The vaccination card was inspected and all vaccinations noted.

\section{Blood sampling}

Blood samples (approximately $600 \mu \mathrm{l}$ ) were taken by finger prick. Blood was sampled into an uncoated tube and a heparinised tube. Additionally, two drops were used to prepare malaria slides and slides for differential leucocyte counts. The blood and the slides were placed in a plastic box protected from sunlight in ambient temperature $\left(28-32^{\circ} \mathrm{C}\right)$ until further processing within maximally $4 \mathrm{~h}$. For detection of malarial infection, thick and thin blood films were prepared on site. The slides were then Giemsa-stained, and microscopically examined for malaria parasites by an experienced laboratory technician.

For analyses of RBP and C-reactive protein (CRP), serum was separated and frozen until analysis. RBP and CRP were measured at SEAMEO, University of Indonesia, Jakarta using a sandwich ELISA technique developed there ${ }^{(11)}$. With regard to vitamin A status, children were classified as sufficient, borderline deficient or deficient using the cut-offs $1 \cdot 11$ and $0 \cdot 83 \mu \mathrm{mol} \mathrm{RBP} / 1$ as described previously ${ }^{(7)}$.

\section{Differential leucocyte counts}

Thin blood smears were prepared on site and air-dried. After methanol fixation, smears were stained using May-GrunwaldGiemsa stains. One observer (I. M. L.) performed all differential leucocyte counts by microscopy, assigning 100 leucocytes to the leucocyte subtypes lymphocyte, monocyte, granulocyte or eosinophil.

\section{In vitro cytokine production}

A whole-blood culture was performed as a 1:10 dilution of heparinised blood in a final volume of $200 \mu \mathrm{l}$ in sterile ninety-six-well plates (Nunc). The dilution was made with glutamine-free Roswell Park Memorial Institute-1640 medium containing streptomycin $(100 \mu \mathrm{g} / \mathrm{ml})$, penicillin A $(100 \mathrm{IU} / \mathrm{ml})$, glutamate ( $2 \mathrm{~mm}$ ) and pyruvate $(1 \mathrm{~mm}$ ) (all Gibco). Stimulations were made in uniplicates performed at conditions leading to submaximal cytokine production using phytohaemagglutinin (PHA, $2 \mu \mathrm{g} / \mathrm{ml}$; Wellcome Diagnostics), lipopolysaccharide (LPS, $100 \mathrm{ng} / \mathrm{ml}$; Sigma-Aldrich Chemie), purified protein derivative of Mycobacterium tuberculosis (PPD, $10 \mu \mathrm{g} / \mathrm{ml}$; Statens Serum Institut) and tetanus toxoid (TT, 1.5 limit-of-flocculation units/ml; NVI). Culture plates were incubated at $37^{\circ} \mathrm{C}$ and $5 \% \mathrm{CO}_{2}$. Supernatants were collected on day 1 (LPS) and day 3 (PPD, PHA and TT). At both days, control samples consisting of diluted blood only were collected (control day 1 and control day 3). Each culture well was assessed at the time of harvesting and any visual signs of contamination were registered. If contamination was suspected in more than three of the six wells, all samples from that child were excluded from the analysis. Individual wells suspected of contamination were not included in the analysis, giving rise to differences in the number of wells analysed for each stimulant. The supernatants were frozen and kept below $-40^{\circ} \mathrm{C}$ until analysis. Supernatant concentrations of IL-5, IL-10, IL-13, IFN- $\gamma$ and TNF- $\alpha$ were measured simultaneously using a Luminex cytokine kit (Luminex Corporation) and a buffer reagent kit (BioSource) on a Luminex-100 cytometer (Luminex Corporation), equipped with StarStation software (Applied Cytometry Systems). The stated 
lower limit of detection (LLD) of the assay was 3, 5, 10, 5 and $10 \mathrm{pg} / \mathrm{ml}$ for IL-5, IL-10, IL-13, IFN- $\gamma$ and TNF- $\alpha$, respectively.

\section{Statistical methods}

Differential leucocyte counts were analysed by multinomial logistic regression, allowing evaluation of the effect of VAS on the overall cell distribution. Multinomial distributions are generalisations of the binomial distribution to more than two outcomes $^{(12)}$. Assignment of 100 independent cells to one of four leucocyte subtypes leads to a multinomial distribution. We used the clustered variance estimator to account for the dependence of having 100 cells from each child. For each subtype, we present the marginal effect of VAS calculated from predictions of the multinomial regression ${ }^{(13)}$. For lymphocytes for example, the marginal effect is the probability that a cell is a lymphocyte in the VAS group minus the probability that a cell is a lymphocyte in the placebo group. Hence, the marginal effect represents the difference in lymphocyte proportion between the VAS and placebo groups.

In the analysis of in vitro cytokine production, samples with a cytokine concentration below the LLD were assigned the value of half the LLD. Data were transformed using the natural logarithm in order to approximate a normal distribution, which was visually confirmed. We conducted two types of analyses:

(1) Log cytokine concentrations were compared by linear regression for cytokines with less than $33 \%$ of samples with non-detectable concentrations (i.e. below the LLD). To account for potential differences in unstimulated cytokine production, we adjusted analyses for the cytokine concentration of the appropriate control, i.e. IL-10 concentrations of day 1 controls were included in the model of LPS-stimulated IL-10 concentrations and IL-10 concentrations of day 3 controls were included in the model of PHA- and PPD-stimulated IL-10 concentrations. The remaining cytokines were treated similarly. Uh et al. ${ }^{(14)}$ have recommended tobit regression as an approach for this kind of cytokine data. Tobit is a censored regression technique for normally distributed data, where the censored observations contribute to the analysis with the probability of being below the censoring limit. We found that results from tobit regression and linear regression were almost identical and chose to present results from the more commonly used linear regression. We calculated the ratios TNF- $\alpha$ :IL-10 and IL-5:IFN- $\boldsymbol{\gamma}$ in order to assess potential effects on the balance of pro- $v$. anti-inflammatory responses and Th2 $v$. Th1 responses, respectively. Log-ratios were analysed by linear regression. Geometric mean ratios (GMR) were obtained by re-transforming all coefficients to the original scale.

(2) For cytokines with more than 33\% of samples with non-detectable concentrations, the cytokine responses were categorised as either detectable ( $>$ LLD) or non-detectable (<LLD). The proportions of detectable responses were compared in Poisson regression models with robust variance estimation, providing relative risks ${ }^{(15)}$.

There was almost no response to TT, neither overall, nor in the $22 \%$ of the children who had received a DTP vaccination before blood sampling. For these reasons, the in vitro response to TT was not included in the analyses.

For investigating the potential correlation between vitamin A status and cytokine production, we used Spearman's rank tests and, additionally, linear regression of the log-transformed cytokine data with vitamin A status as a continuous variable.

A significance level of $5 \%$ was used for all analyses with the exception of interactions for which a $P$ value $<0 \cdot 1$ was interpreted as significant. All analyses were done using Stata/SE 10 (Statacorp LP).

\section{Ethical considerations}

The trial, including the immunological subgroup study, was conducted according to the guidelines of the Declaration of Helsinki and all procedures were approved by the Ministry of Health in Guinea-Bissau and by the Danish Central Ethical Committee. The trial was registered at ClinicalTrials.gov as NCT00168597. For all subjects, verbal informed consent was obtained, witnessed and formally recorded.

\section{Results}

A total of 480 children were included in the immunological subgroup study. Of these, nine were older than 3 months at sampling and were excluded for this reason. The basic characteristics of the included children by intervention group are presented in Table 1 . With regard to the in vitro cytokine production, ten children had signs of contamination and were excluded; hence, results were available for 461 children. A significantly larger proportion of samples obtained from children in the VAS arm was excluded due to contamination (9) v. $1, P<0 \cdot 01, \chi^{2}$ test). Results from differential leucocyte counts were available for 467 children.

\section{Cell distributions}

Tables 2, 3 and 4 shows the distribution of cell subtypes from the differential leucocyte count and the corresponding differences between the treatment groups. Overall, the distribution of the four subtypes differed between the VAS and placebo groups ( $P=0 \cdot 04$; Table 2 ). This was due to a higher frequency of monocytes among the VAS recipients (difference 0.9, 95\% CI $0.3,1.5$ ). When data were stratified by sex (Table 3 ), the higher frequency of monocytes after VAS was mainly observed among girls (difference 1.2, 95\% CI 0.4, 2.1), whereas there was no effect of VAS among boys. However, the test for interaction between sex and VAS was not significant $(P$ for interaction 0.58). Stratification by DTP status at the time of blood sampling (Table 4) showed that the higher monocyte frequency among children who received VAS was significant only among the DTP recipients (difference $1 \cdot 7$, 95\% CI $0 \cdot 6,2 \cdot 9)$, but with no significant interaction between DTP 
Table 2. Effect of vitamin A supplementation (VAS) on leucocyte subtype frequency for all subjects†

(Mean values and standard deviations)

\begin{tabular}{lrrrrrrr}
\hline & \multicolumn{2}{c}{ VAS $(n$ 221 $)$} & & \multicolumn{2}{c}{ Placebo $(n$ 246) } \\
\cline { 2 - 3 } Cell subtype & Mean & SD & & Mean & SD & Difference* $^{*}$ & $95 \%$ Cl \\
\hline Lymphocytes & 67.0 & 11.9 & & 67.5 & 11.1 & -0.5 & $-2.6,1.6$ \\
Granulocytes & 25.3 & 10.9 & & 25.8 & 10.4 & -0.5 & $-2.4,1.6$ \\
Eosinophils & 3.0 & 2.7 & & 2.9 & 3.2 & 0.1 & $-0.4,0.6$ \\
Monocytes & 4.7 & 3.6 & & 3.8 & 3.3 & 0.9 & $0.3,1.5$
\end{tabular}

* Statistically significant overall effect of VAS on leucocyte distribution; $P=0.04$.

†Crude estimates resulting from multiple logistic regression without adjustments. Estimates and

$95 \% \mathrm{Cl}$ for differences between treatment groups are derived using average marginal effects.

status and VAS ( $P$ for interaction $0 \cdot 21$ ). $P$ for the for three-way interaction between VAS, sex and DTP status was 0.94.

\section{In vitro cytokine production}

There was no overall effect of VAS on the stimulated production of any of the included cytokines (Table 5). There was a highly significant effect of VAS on spontaneous TNF- $\alpha$ production on day 1 after sampling, as seen by the lower cytokine concentrations in the control wells on day 1 (Table 5). VAS recipients had almost half the concentrations of TNF- $\alpha$ than children receiving placebo. The difference remained if we adjusted for being ill or included CRP concentrations in the model (GMR adjusted for being ill: 0.54, 95\% CI 0.35, $0.73, P<0.001$; GMR adjusted for CRP: $0.49,95 \%$ CI 0.34 $0 \cdot 71, P<0 \cdot 001)$.

The effect of VAS on spontaneous TNF- $\alpha$ production was significant for girls (GMR 0.43, 95\% CI 0.26, 0.72) but not for boys (GMR $0 \cdot 70$, 95\% CI $0.41,1 \cdot 20$ ) ( $P$ for interaction $0 \cdot 21)$. In addition, we observed significant interactions between sex and VAS with regard to TNF- $\alpha$ production in response to LPS and PHA stimulation. Boys receiving VAS had a smaller TNF- $\alpha$ response to LPS compared with boys receiving placebo (GMR 0.68, 95\% CI 0.47, 1.00, $P=0.05$ ), while girls displayed no effect (GMR 1.09, 95\% CI 0.76, 1.56, $P=0.64$ ) ( $P$ for interaction 0.08 ). For the PHA stimulation, the opposite was seen with a tendency for higher TNF- $\alpha$ production among the male VAS recipients compared with placebo (GMR 1.38, 95\% CI 0.95, 1.99, $P=0.09$ ). Again, no effect was seen among girls (GMR 0.89, 95\% CI 0.63, 1.27, $P=0.53 ; P$ for interaction 0.096). Results were similar when conducting the analysis only on children not receiving DTP before blood sampling ( $n$ 358), and there were no significant interactions between VAS and DTP (data not shown).

We found a borderline significant effect of receiving VAS on the TNF- $\alpha$ :IL-10 ratio in unstimulated blood on day 1 ; children who had received VAS had lower ratios (GMR 0.76, 95\% CI $0.57,1.01, P=0.06)$. A similar pattern was seen for the response to LPS (GMR (unadjusted) 0.78, $95 \%$ CI 0.59, 1.04, $P=0.09$ ). No significant effect of VAS was seen for PHA or PPD or when we calculated the Th2:Th1 ratios (IL-5:IFN- $\gamma$; data not shown).

When the results were stratified by sex and DTP status, lower spontaneous TNF- $\alpha$ production on day 1 among the VAS recipients persisted among boys who had received DTP (GMR 0.26, 95\% CI 0.08, 0.81) and among girls who had not received DTP (GMR 0.37, 95\% CI 0.21, 0.67) (Table 6 and Fig. 1). This resulted in a significant three-way interaction between receiving VAS, DTP status and sex $(P$ for interaction 0.03). In parallel, the concentration of IL-10 seemed to be dependent on the combination of VAS, DTP status and sex (Table 6 and Fig. 1): girls who had not received DTP had a significantly lower spontaneous IL-10 production on day 1 (GMR $0.49,95 \%$ CI $0.27,0.87)$ after VAS. Boys who had received DTP had the same but non-significant tendency towards lower IL-10, while boys who had not received DTP appeared to be unaffected. No effect was seen among girls receiving DTP. Overall, this resulted in a significant three-way

Table 3. Effect of vitamin A supplementation (VAS) on leucocyte subtype frequency by sexł‡

(Mean values and standard deviations)

\begin{tabular}{|c|c|c|c|c|c|c|c|c|c|c|c|c|}
\hline \multirow[b]{2}{*}{ Cell subtype } & \multicolumn{6}{|c|}{ Boys } & \multicolumn{6}{|c|}{ Girls } \\
\hline & Mean & SD & Mean & SD & Difference & $95 \% \mathrm{Cl}$ & Mean & SD & Mean & SD & Difference* & $95 \% \mathrm{Cl}$ \\
\hline Lymphocytes & $68 \cdot 1$ & 11.3 & $67 \cdot 6$ & $10 \cdot 3$ & 0.5 & $-2 \cdot 3,3 \cdot 4$ & $66 \cdot 0$ & $12 \cdot 3$ & $67 \cdot 4$ & 11.8 & -1.4 & $-4.4,1.7$ \\
\hline Granulocytes & 24.6 & $10 \cdot 7$ & $25 \cdot 6$ & $10 \cdot 0$ & -1.0 & $-3 \cdot 7,1 \cdot 7$ & 25.9 & $11 \cdot 1$ & $26 \cdot 0$ & $10 \cdot 8$ & -0.1 & $-2 \cdot 8,2 \cdot 7$ \\
\hline Eosinophils & $2 \cdot 7$ & $2 \cdot 8$ & $2 \cdot 8$ & 3.3 & -0.1 & $-0.9,0.7$ & $3 \cdot 2$ & $2 \cdot 6$ & 3.0 & 3.2 & 0.2 & $-0.5,0.9$ \\
\hline Monocytes & 4.6 & 3.6 & $4 \cdot 1$ & 3.5 & 0.5 & $-0.4,1.5$ & 4.8 & 3.6 & 3.6 & $3 \cdot 1$ & 1.2 & $0.4,2 \cdot 1$ \\
\hline
\end{tabular}


Table 4. Effect of vitamin A supplementation (VAS) on leucocyte subtype frequency by whether or not the diphtheria-tetanus-pertussis (DTP) vaccine had been received prior to blood sampling $\dagger \ddagger$

(Mean values and standard deviations)

\begin{tabular}{|c|c|c|c|c|c|c|c|c|c|c|c|c|}
\hline \multirow[b]{3}{*}{ Cell subtype } & \multicolumn{6}{|c|}{ DTP } & \multicolumn{6}{|c|}{ No DTP } \\
\hline & \multicolumn{2}{|c|}{ VAS $(n 56)$} & \multicolumn{2}{|c|}{ Placebo ( $n$ 48) } & \multirow[b]{2}{*}{ Difference* } & \multirow[b]{2}{*}{$95 \% \mathrm{Cl}$} & \multicolumn{2}{|c|}{ VAS $(n 165)$} & \multicolumn{2}{|c|}{ Placebo ( $n$ 125) } & \multirow[b]{2}{*}{ Difference } & \multirow[b]{2}{*}{$95 \% \mathrm{Cl}$} \\
\hline & Mean & SD & Mean & SD & & & Mean & SD & Mean & SD & & \\
\hline Lymphocytes & 62.5 & $12 \cdot 7$ & $66 \cdot 7$ & $12 \cdot 2$ & -4.2 & $-9.0,0.6$ & 68.5 & 11.3 & $67 \cdot 4$ & $10 \cdot 8$ & 0.8 & $-1 \cdot 4,3 \cdot 1$ \\
\hline Granulocytes & $29 \cdot 0$ & 11.9 & $27 \cdot 1$ & 11.4 & 1.9 & $-2.5,6.4$ & $24 \cdot 1$ & $10 \cdot 3$ & 25.5 & $10 \cdot 2$ & -1.4 & $-3 \cdot 6,0.7$ \\
\hline Eosinophils & $2 \cdot 9$ & $3 \cdot 1$ & 2.4 & $2 \cdot 7$ & 0.6 & $-0.6,1.7$ & $3 \cdot 0$ & $2 \cdot 5$ & 3.0 & 3.3 & 0.0 & $-0.6,0.6$ \\
\hline Monocytes & $5 \cdot 6$ & 3.2 & 3.8 & 2.9 & 1.7 & $0.6,2.9$ & 4.4 & 3.7 & 3.8 & 3.4 & 0.6 & $-0.1,1.4$ \\
\hline
\end{tabular}

* Statistically significant overall effect of VAS on leucocyte distribution for having received DTP; $P=0.03$. For not having received DTP, the overall effect was NS; $P=0.23$.

†Test for interaction between VAS and DTP status; $P=0.21$. Test for three-way interaction: VAS, sex, DTP status: $P=0.94$.

$\ddagger$ Crude estimates resulting from multiple logistic regression without adjustments. Estimates and $95 \% \mathrm{Cl}$ for differences between treatment groups are derived using average marginal effects.

interaction between VAS, sex and DTP ( $P$ for interaction $0 \cdot 04$ ). We also noted that PHA-stimulated IL-5 production was significantly affected by VAS among boys who had received DTP: VAS recipients in this strata displayed significantly higher IL-5 concentrations than placebo recipients (GMR 2.56, $95 \%$ CI 1.10, 5.99). Among boys who had received DTP, the IL-13 response to PPD was also higher after VAS than after placebo (GMR 2.25, $95 \%$ CI 1·18, 4.32). On average, DTP recipients were older than children who did not receive
DTP. However, adjusting the analyses for age had essentially no effect on the estimates.

For cytokines where more than one-third of the samples were below the LLD, we examined the risk of having a detectable cytokine response. Using this approach on the unstratified data, we observed no statistically significant effects of VAS for any of the cytokines. When we performed the analysis stratified by sex, we found an interaction between VAS and sex for spontaneous IFN- $\gamma$ production on day 3

Table 5. Effect of vitamin A supplementation (VAS) on in vitro cytokine production $†$

(Geometric means (GM) and 95\% confidence intervals)

\begin{tabular}{|c|c|c|c|c|c|c|c|c|}
\hline & \multicolumn{3}{|c|}{ VAS $(n 214)$} & \multicolumn{3}{|c|}{ Placebo ( $n$ 247) } & \multirow[b]{2}{*}{ GMR or RR } & \multirow[b]{2}{*}{$95 \% \mathrm{Cl}$} \\
\hline & GM (pg/ml) & $95 \% \mathrm{Cl}$ & $\%$ ND & GM (pg/ml) & $95 \% \mathrm{Cl}$ & $\%$ ND & & \\
\hline \multicolumn{9}{|l|}{ TNF- $\alpha$} \\
\hline Control day 1 & 487 & 364,653 & $5 \cdot 2$ & 901 & 711,1142 & $2 \cdot 0$ & $0.54^{*}$ & $0.37,0.78$ \\
\hline LPS & 7507 & 6004,9386 & $3 \cdot 3$ & 9857 & 8389,11583 & $1 \cdot 2$ & 0.87 & $0.67,1.13$ \\
\hline Control day 3 & 112 & 83,152 & $15 \cdot 6$ & 149 & 111,199 & $14 \cdot 2$ & 0.75 & $0.50,1.15$ \\
\hline PHA & 871 & 719,1055 & $2 \cdot 8$ & 848 & 694, 1037 & $3 \cdot 7$ & $1 \cdot 11$ & $0.86,1.44$ \\
\hline PPD & 726 & 564,935 & $6 \cdot 7$ & 892 & 696,1142 & $6 \cdot 0$ & 0.89 & $0.64,1.24$ \\
\hline \multicolumn{9}{|l|}{ IL-10 } \\
\hline Control day 1 & 32 & 24,42 & 26.9 & 45 & 35,58 & $20 \cdot 7$ & 0.71 & $0.49,1.04$ \\
\hline LPS & 917 & 810,1038 & 0.0 & 942 & 833,1066 & 0.4 & 1.06 & $0.92,1.22$ \\
\hline Control day 3 & 12 & 10,16 & $48 \cdot 2$ & 17 & 13,22 & $40 \cdot 3$ & $0.87 \ddagger$ & $0.73,1.03$ \\
\hline PHA & 120 & 102,140 & 1.9 & 122 & 104,143 & $2 \cdot 1$ & 1.09 & $090,1.32$ \\
\hline PPD & 66 & 55,80 & $5 \cdot 3$ & 73 & 60,90 & $7 \cdot 8$ & 1.07 & $0.89,1.30$ \\
\hline \multicolumn{9}{|l|}{ IL-5 } \\
\hline Control day 3 & 2 & 2,2 & $90 \cdot 0$ & 2 & 2,2 & $86 \cdot 3$ & $0.73 \ddagger$ & $0.43,1.24$ \\
\hline PHA & 121 & 98,149 & $2 \cdot 4$ & 99 & 81,121 & $4 \cdot 1$ & 1.23 & $0.92,1.64$ \\
\hline PPD & 13 & 11,17 & $26 \cdot 8$ & 12 & 10,15 & $24 \cdot 1$ & 1.09 & $0.81,1.46$ \\
\hline \multicolumn{9}{|l|}{ IL-13 } \\
\hline Control day 3 & 14 & 12,17 & $50 \cdot 3$ & 13 & 11,15 & 50.0 & $0.99 \ddagger$ & $0.82,1.20$ \\
\hline PHA & 342 & 294, 399 & $3 \cdot 3$ & 324 & 284,370 & 2.9 & 1.05 & $0.86,1.29$ \\
\hline PPD & 63 & 55,74 & 11.5 & 51 & 44,60 & $16 \cdot 8$ & 1.22 & $0.98,1.53$ \\
\hline \multicolumn{9}{|l|}{ IFN- $\gamma$} \\
\hline Control day 3 & 6 & 5,7 & $58 \cdot 8$ & 6 & 5,7 & 54.4 & $0.90 \ddagger$ & $0.73,1.13$ \\
\hline PHA & 138 & 113,169 & $2 \cdot 8$ & 126 & 104,154 & 2.5 & $1 \cdot 12$ & $0.85,1.48$ \\
\hline PPD & 170 & 133,219 & $5 \cdot 3$ & 136 & 106,174 & $7 \cdot 8$ & 1.29 & $0.91,1.83$ \\
\hline
\end{tabular}


Table 6. Effect of vitamin A supplementation (VAS) on cytokine production by sex and having received the diphtheria-tetanus-pertussis (DTP) vaccine† (Geometric means and $95 \%$ confidence intervals)

\begin{tabular}{|c|c|c|c|c|c|c|c|c|c|c|c|c|c|c|c|c|c|}
\hline & \multicolumn{8}{|c|}{ Boys } & \multicolumn{8}{|c|}{ Girls } & \multirow{4}{*}{$\begin{array}{c}P \text { for } \\
\text { three-way } \\
\text { interaction } \\
\text { between } \\
\text { VAS, sex } \\
\text { and DTP }\end{array}$} \\
\hline & \multicolumn{4}{|c|}{ DTP prior to blood sampling ( $n$ 49) } & \multicolumn{4}{|c|}{$\begin{array}{l}\text { No DTP prior to blood } \\
\text { sampling }(n 170)\end{array}$} & \multicolumn{4}{|c|}{ DTP prior to blood sampling ( $n 54)$} & \multicolumn{4}{|c|}{$\begin{array}{l}\text { No DTP prior to blood } \\
\text { sampling }(n 188)\end{array}$} & \\
\hline & \multicolumn{2}{|c|}{$\begin{array}{c}\text { Geometric } \\
\text { mean }(p g / m l)\end{array}$} & \multirow{2}{*}{$\begin{array}{l}\text { GMR } \\
\text { or RR }\end{array}$} & \multirow[b]{2}{*}{$95 \% \mathrm{Cl}$} & \multicolumn{2}{|c|}{$\begin{array}{c}\text { Geometric } \\
\text { mean }(p g / m l)\end{array}$} & \multirow{2}{*}{$\begin{array}{l}\text { GMR } \\
\text { or RR }\end{array}$} & \multirow[b]{2}{*}{$95 \% \mathrm{Cl}$} & \multicolumn{2}{|c|}{$\begin{array}{c}\text { Geometric } \\
\text { mean }(\mathrm{pg} / \mathrm{ml})\end{array}$} & \multirow{2}{*}{$\begin{array}{l}\text { GMR } \\
\text { or RR }\end{array}$} & \multirow[b]{2}{*}{$95 \% \mathrm{Cl}$} & \multicolumn{2}{|c|}{$\begin{array}{c}\text { Geometric } \\
\text { mean }(\mathrm{pg} / \mathrm{ml})\end{array}$} & \multirow{2}{*}{$\begin{array}{l}\text { GMR } \\
\text { or RR }\end{array}$} & \multirow[b]{2}{*}{$95 \% \mathrm{Cl}$} & \\
\hline & VAS & Placebo & & & VAS & Placebo & & & VAS & Placebo & & & VAS & Placebo & & & \\
\hline \multicolumn{18}{|l|}{ TNF- $\alpha$} \\
\hline Control Day 1 & 361 & 1375 & $0.26^{*}$ & $0.08,0.81$ & 728 & 777 & 0.94 & $0.53,1.67$ & 555 & 766 & 0.72 & $0.19,2.72$ & 361 & 976 & $0.37^{\star}$ & $0.21,0.67$ & $0.03^{*}$ \\
\hline LPS & 5395 & 14932 & 0.45 & $0.16,1 \cdot 30$ & 7996 & 10656 & 0.76 & $0.48,1.19$ & 8027 & 10857 & 0.79 & $0.48,1.29$ & 7652 & 8090 & $1 \cdot 17$ & $0.78,1.77$ & 0.93 \\
\hline Control Day 3 & 88 & 163 & 0.54 & $0 \cdot 14,2 \cdot 17$ & 135 & 136 & 0.99 & $0.49,2.02$ & 151 & 105 & 1.44 & $0.42,4.93$ & 93 & 172 & 0.54 & $0.28,1.04$ & 0.12 \\
\hline PHA & 1017 & 635 & 1.86 & $0.71,4.87$ & 951 & 732 & 1.30 & $0.85,2.00$ & 915 & 837 & 0.98 & $0.52,1.86$ & 761 & 1040 & 0.87 & $0.59,1.29$ & 0.77 \\
\hline PPD & 517 & 694 & 0.73 & $0.20,2 \cdot 62$ & 764 & 932 & 0.84 & $0.48,1.46$ & 895 & 725 & 0.99 & $0.49,2.02$ & 709 & 955 & 0.91 & $0.55,1.52$ & 0.90 \\
\hline \multicolumn{18}{|l|}{ IL-10 } \\
\hline Control Day 1 & 23 & 54 & 0.42 & $0.14,1.33$ & 34 & 35 & 0.99 & $0.52,1.89$ & 53 & 39 & 1.36 & $0.43,4.36$ & 28 & 56 & $0.49^{*}$ & $0.27,0.87$ & $0.04^{*}$ \\
\hline LPS & 735 & 936 & 0.96 & $0.69,1.34$ & 1005 & 914 & 1.09 & $0.85,1.38$ & 841 & 663 & 1.14 & $0.72,1.81$ & 933 & 1056 & 1.06 & $0.84,1.33$ & 0.58 \\
\hline Control Day $3 \ddagger$ & $65 \cdot 2$ & $33 \cdot 3$ & $0.52^{\star}$ & $0.28,0.98$ & $47 \cdot 8$ & $46 \cdot 6$ & 0.98 & $0.72,1.32$ & $39 \cdot 3$ & $45 \cdot 5$ & 1.11 & $0.68,1.82$ & $46 \cdot 9$ & $34 \cdot 8$ & 0.81 & $0.63,1.05$ & $0.04^{*}$ \\
\hline PHA & 66 & 63 & 1.26 & $0.67,2.37$ & 134 & 113 & $1 \cdot 19$ & $0.84,1.68$ & 94 & 124 & 0.77 & $0.42,1.43$ & 141 & 151 & $1 \cdot 14$ & $0.89,1.46$ & 0.37 \\
\hline PPD & 29 & 47 & 0.88 & $0.46,1.66$ & 81 & 67 & 1.24 & $0.93,1.66$ & 59 & 51 & $1 \cdot 10$ & $0.59,2.06$ & 73 & 97 & 1.05 & $0.79,1.40$ & 0.34 \\
\hline \multicolumn{18}{|l|}{ IL-5 } \\
\hline Control Day 3‡ & 95.7 & 83.4 & 0.26 & $0.03,2.21$ & 91.0 & $83 \cdot 0$ & 0.53 & $0.21,1.29$ & 92.7 & 95.5 & 1.57 & $0 \cdot 15,16 \cdot 61$ & 86.4 & 88.0 & $1 \cdot 14$ & $0.52,2.48$ & 0.55 \\
\hline PHA & 194 & 76 & 2.56 & $1 \cdot 10,5.99$ & 122 & 97 & 1.29 & $0 \cdot 76,2 \cdot 20$ & 96 & 112 & 0.85 & $0.39,1.82$ & 114 & 105 & 1.07 & $0.68,1.67$ & 0.19 \\
\hline PPD & 20 & 17 & 1.34 & $0.52,3.46$ & 14 & 11 & 1.30 & $0 \cdot 78,2 \cdot 16$ & 33.3 & 33.3 & 1.02 & $0.69,1.52$ & 12 & 13 & 0.88 & $0.56,1.38$ & 0.90 \\
\hline \multicolumn{18}{|l|}{ IL-13 } \\
\hline Control Day $3 \ddagger$ & $52 \cdot 0$ & $45 \cdot 8$ & 0.88 & $0.50,1.56$ & 47.8 & $51 \cdot 1$ & 1.07 & $0.78,1.46$ & $57 \cdot 1$ & 36.4 & 0.67 & $0.39,1.15$ & 49.4 & $53 \cdot 3$ & 1.08 & $0.80,1.47$ & 0.53 \\
\hline PHA & 440 & 250 & 1.78 & $0.97,3.27$ & 306 & 328 & 0.93 & $0.65,1.33$ & 345 & 373 & 0.96 & $0.56,1.63$ & 350 & 329 & 1.05 & $0.76,1.45$ & 0.13 \\
\hline PPD & 105 & 46 & $2 \cdot 25^{\star}$ & $1 \cdot 18,4.32$ & 63 & 51 & 1.22 & $0.85,1.74$ & 58 & 47 & 1.34 & $0.71,2.53$ & 58 & 54 & 1.06 & $0.73,1.54$ & 0.46 \\
\hline \multicolumn{18}{|l|}{ IFN- $\gamma$} \\
\hline Control Day 3‡ & $65 \cdot 2$ & 62.5 & 0.92 & $0.43,2.00$ & $49 \cdot 3$ & 58.0 & $1 \cdot 21$ & $0.86,1.70$ & 67.9 & $50 \cdot 0$ & 0.64 & $0.32,1 \cdot 27$ & $61 \cdot 7$ & $50 \cdot 0$ & 0.77 & $0.54,1.08$ & 0.88 \\
\hline PHA & 245 & 176 & 1.40 & $0.51,3.81$ & 118 & 118 & 0.98 & $0.61,1.58$ & 132 & 103 & $1 \cdot 14$ & $0.55,2.37$ & 136 & 131 & $1 \cdot 14$ & $0.75,1.73$ & 0.82 \\
\hline PPD & 333 & 195 & 1.70 & $0.41,7.09$ & 163 & 124 & 1.29 & $0.71,2.32$ & 185 & 139 & 1.32 & $0.56,3.14$ & 143 & 135 & $1 \cdot 18$ & $0.69,2.01$ & 0.94 \\
\hline
\end{tabular}

GMR, geometric mean ratio; RR, relative risk; LPS, lipopolysaccharide; PHA, phytohaemagglutinin; PPD, purified protein derivative of Mycobacterium tuberculosis; \%ND, percentage of samples under the limit of detection. * Statistically significant findings.

†Analysed using linear regression of log-transformed cytokine values. For stimulated responses the spontaneous cytokine production was taken into account by adjusting for the cytokine concentration of the control. † Linear regression not performed due to too high proportion of non-detectable samples. \%ND displayed instead of geometric mean and the estimate displayed is instead from crude Poisson regression reflecting relative risk of
having a detectable sample. 
(a)

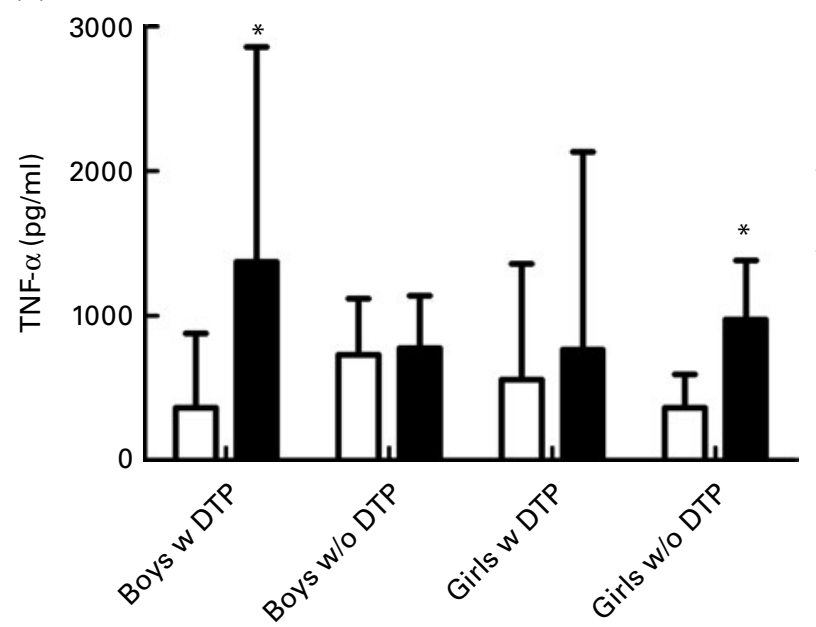

(b)

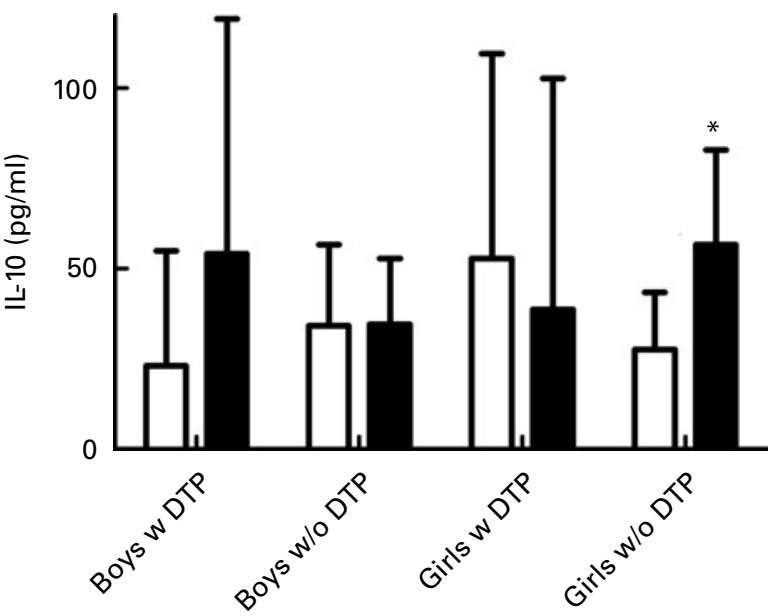

(c)

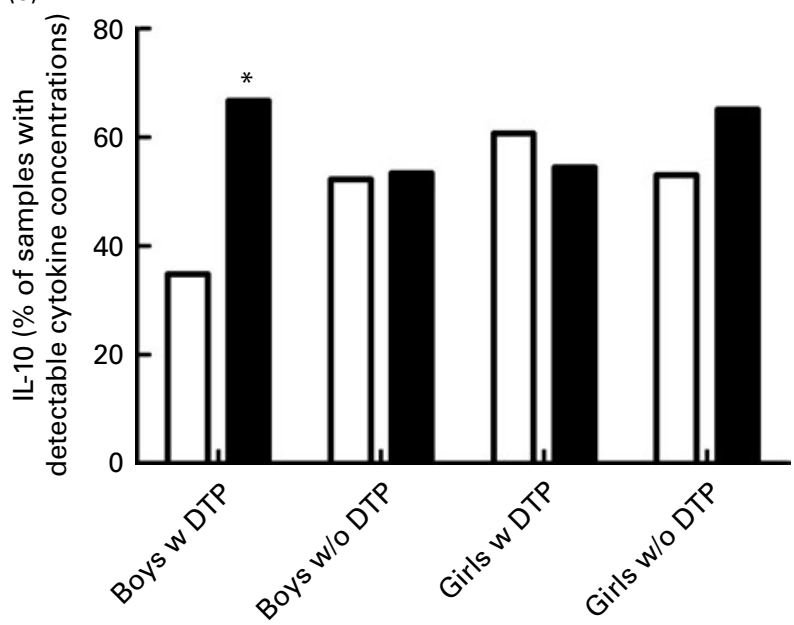

Fig. 1. Unstimulated TNF- $\alpha$ and IL-10 production (geometric means and $95 \%$ confidence intervals) for vitamin A supplementation (VAS) and placebo stratified by sex and diphtheria-tetanus-pertussis (DTP) vaccination status. (a) TNF- $\alpha$-control day 1. $P$ for three-way interaction between sex, DTP and VAS: 0.03 . (b) IL-10control day 1. $P$ for three-way interaction between sex, DTP and VAS: 0.04 . (c) IL-10-control day 3. $P$ for three-way interaction between sex, DTP and VAS: 0.04. ${ }^{*}$ Mean value was significantly different from that of the placebo group $(P<0.05)$. $\square$, VAS; $\square$, placebo; w, with; w/o, without.

( $P$ for interaction 0.05). Among girls, few VAS recipients had detectable responses compared with placebo recipients (relative risk $0.73,95 \%$ CI $0.54,0.99$ ), whereas the opposite tendency was seen among boys (relative risk 1.13, 95\% CI $0.83,1.55)$. The effect of VAS was the same irrespective of DTP status (data not shown). However, stratified by sex and DTP, there was a significant three-way interaction on the risk of having a detectable concentration of spontaneous IL-10 production on day 3 ( $P$ for interaction $0 \cdot 04$; Table 6 ). Among DTP-vaccinated boys, few VAS recipients had detectable concentrations compared with placebo recipients. Among girls not receiving DTP, we noted the opposite tendency. There was no apparent effect of VAS among boys who did not receive DTP and girls who had received DTP.

\section{Effect of vitamin A status on cytokine concentrations}

Using the categories sufficient, borderline deficient and deficient for vitamin A status in Spearman's rank analysis, we found that vitamin A status was positively correlated with IL-5 and IL-13 production to PPD $(r 0 \cdot 11 ; P=0.04$ and $r 0.12 ; P=0.03$, respectively) if excluding children with ongoing inflammation (ninety-eight out of 434 excluded). For none of the other cytokines we noted any correlations (data not shown).

\section{Discussion}

We investigated the effect of VAS at birth on the immune system 6 weeks later through differential leucocyte counts and an evaluation of the in vitro response to a panel of antigens/mitogens using a whole-blood culture protocol. Children receiving VAS had higher levels of monocytes in the peripheral blood but lower spontaneous production of TNF- $\alpha$. None of the stimulants showed differences between the VAS and placebo groups. However, stratified post hoc analyses revealed that the effect of VAS on some cytokine concentrations may have differed by sex and depending on whether the child had received DTP or not at the time of blood sampling. 
Limitations of the present study are inherent in its explorative nature: many analyses were carried out and the risk of chance findings is high. Hence, the results should be interpreted with great caution and mainly as hypothesis generating. The absence of a pre-intervention measurement in the present study means that inherent differences between treatment groups could influence the results. However, since the present study was nested in a randomised trial, these potential confounders are likely to be distributed evenly between treatment groups.

With this in mind, one interpretation of lower spontaneous TNF- $\alpha$ production after VAS could be that VAS recipients had few infections or that infections were less severe due to an improvement in immune function. For placebo recipients, infections could thus translate to a higher spontaneous production of pro-inflammatory $\mathrm{TNF}-\alpha$ due to a lowered threshold to external stimuli per se or an activated state of macrophages/monocytes. However, lower TNF- $\alpha$ concentration among the VAS recipients persisted even after adjusting for CRP and being ill. This could suggest that the present finding does not reflect differences in healthy state at blood sampling between the two groups, but corresponds to differences at the steady state of a resting immune system. We speculate that these differences may not be evident when observing the stimulated immune response. In one study utilising human cord blood peripheral mononuclear cells as well as a monocyte/macrophage cell line, it was found that in vitro treatment of the cells with the bioactive vitamin A metabolite all-trans-retinoic acid (atRA) resulted in increased IL-10 and decreased TNF- $\alpha$ production after stimulation ${ }^{(16)}$. Another study assessed peritoneal macrophage function in rats and showed a dose-dependent decrease in LPS-stimulated TNF- $\alpha$ production with in vitro atRA treatment ${ }^{(17)}$. To our knowledge, the present study is the first to show an effect of vitamin A on baseline TNF- $\alpha$ production in human subjects. We find it noteworthy that VAS may induce differences despite no apparent effect on vitamin A status. We interpret this as indicative that VAS can have immunomodulatory effects irrespective of pre-existing vitamin A deficiency in parallel with murine experiments showing an impact on the antibody response after oral atRA ${ }^{(18)}$.

It is a popular, although disputed ${ }^{(19-21)}$, point of view that vitamin $\mathrm{A}$ and related metabolites hold anti-inflammatory potential especially decreasing Th1 responses, thus skewing the immune response in a Th2 direction ${ }^{(22)}$. VAS may have reduced Th1 skewing in girls, as witnessed by the significantly reduced proportion of girls with detectable spontaneous IFN- $\gamma$ levels. There was no effect of VAS on Th2 skewing overall. However, in the stratified analyses, VAS was associated with a higher IL-5 response to PHA and IL-13 to PPD among boys receiving DTP, consistent with a Th2 skewing. Thus, although our study does not confirm a generalised Th2-skewing effect of VAS, it is noteworthy that the results of the subgroup analyses are consistent with previous reports, at least for boys receiving DTP.

We observed that VAS recipients had a higher fraction of monocytes. As monocytes are considered to be the main producers of $\mathrm{TNF}-\alpha^{(23)}$, it is interesting to note the apparently contradictive observations that VAS was associated with a larger monocyte fraction but lower TNF- $\alpha$ production. One could speculate that the individual monocyte is producing less TNF- $\alpha$ at the steady-state level. However, we do not have an absolute leucocyte count, and cannot exclude that VAS recipients had less circulating leucocytes and thereby a lower absolute number of monocytes. Monocytes are, however, not the sole TNF- $\alpha$ producers, and quantitative and/or qualitative differences of other cell types such as $\mathrm{T}$ lymphocytes could also be the source of decreased TNF- $\alpha$ production after VAS. Very few have studied the effect of VAS on leucocyte counts but one animal study has reported increases in total leucocyte numbers in response to an intensive regimen involving daily injections of atRA, for $5 \mathrm{~d}^{(24)}$. A study of the effect of VAS as a treatment in measles infection in children reported an increase in total lymphocyte numbers in vitamin A-treated children but no data on the total leucocyte number were provided $^{(25)}$. Ueki et al. ${ }^{(26)}$ have reported that retinoic acids at physiological concentrations can inhibit eosinophil apoptosis in vitro and although the study was concerned with immediate effects of retinoic acid, it might lead one to expect that eosinophil counts would display a difference between groups in the present study. We did not observe any positive association between VAS and eosinophil proportion, but this does not exclude that the absolute number of eosinophils might have been higher in VAS recipients.

A previous publication from our group including, in part, the same participants as the present study investigated the effect of VAS on the immune response to the BCG vaccine ${ }^{(10)}$ and found that VAS was associated with a significantly increased net IFN- $\gamma$ response to PPD. This tendency was seen in the present study, but it did not reach statistical significance.

A recent publication showed an inverse correlation between whole-body vitamin A stores and LPS-induced IL-10 production in a whole-blood set-up very similar to the present study, although addressing adult immune function ${ }^{(27)}$. We tested that observation in our data but did not find any correlation between IL-10 production and vitamin A status assessed by serum RBP concentrations.

The present study was nested in a randomised controlled trial, which has reported that the effect of VAS at birth was modified by sex and receiving DTP. Prompted by these results, we conducted a stratified analysis in the present dataset. With regard to the differential leucocyte counts, we observed that the positive association between VAS and monocyte proportion was particularly evident for girls and for children who had received DTP. The in vitro cytokine production showed a differential effect of VAS by sex and DTP status on spontaneous TNF- $\alpha$ production and IL-10 production on day 1 and spontaneous IL-10 production on day 3. As illustrated in Fig. 1, in all three instances, receiving VAS was associated with lower cytokine production for boys who had received DTP and girls who had not received DTP. In contrast, there was no effect of VAS among boys not receiving DTP and among girls receiving DTP.

Can these results be interpreted in light of the observed mortality pattern with an increase in mortality among female 
VAS recipients after DTP and no apparent effect among boys? The present in vitro findings corroborate that health interventions do not necessarily affect boys and girls similarly. We speculate that the reason might be underlying differences in the immune system between the two sexes. In parallel, one may speculate that sex-differential responses to the same infectious insult can occur. A recent study seems to support this line of thought, reporting that inflammatory markers, CRP, lymphocyte and neutrophil counts were different for hospitalised boys and girls, overall and when stratified according to the reason for hospitalisation ${ }^{(28)}$. The putative existence of sex-differential immune responses means that we should use even more caution than usual, when translating in vitro findings into real-life situations.

In summary, we found that children receiving VAS at birth had a higher proportion of monocytes. In line with other studies, which have observed that vitamin A has antiinflammatory capacities, VAS at birth decreased spontaneous TNF- $\alpha$ production in a whole-blood culture set-up. The present results indicate that VAS at birth affects the resting state of the immune system measured 6 weeks later and that the extent of this basic immune modulation could be dependent on other factors such as sex and DTP status. The results seem to substantiate the potential role of VAS as an immunomodulatory intervention in addition to its role in relieving vitamin A deficiency. An elucidation of the underlying mechanisms of the effects of VAS holds great potential for further improving the chance that more children in low-income countries will be getting their vitamin $\mathrm{A}$ in the right dose at the right time.

\section{Acknowledgements}

The authors wish to acknowledge the work of Amabelia Rodrigues for her involvement in the design of the main study, as well as the contribution of Juergen Erhardt regarding the RBP/CRP measurements. This study was supported by the European Commission, International Cooperation (INCO) programme (contract no. ICA4-CT-2002-10053), the Danish Medical Research Council, the March of Dimes, the University of Copenhagen and the Ville Heise Foundation. The Bandim Health Project received support from the Danish International Development Agency (DANIDA) and the Danish National Research Foundation. P. A. holds a research professorship grant from the Novo Nordisk Foundation. None of the authors has any conflicts of interest. C. S. B., P. A. and M. Y. designed the study; C. S. B., P. A., I. M. L., A. B. F., M. Y. and E. S. conducted the research; A. A. provided statistical support; M. J. J. analysed the data and performed the statistical analysis; M. J. J. wrote the first draft of the manuscript. All authors read and approved the final manuscript.

\section{References}

1. Stephensen CB (2001) Vitamin A, infection, and immune function. Annu Rev Nutr 21, 167-192.

2. Benn CS, Diness BR, Roth A, et al. (2008) Effect of 50,000 IU vitamin A given with BCG vaccine on mortality in infants in
Guinea-Bissau: randomised placebo controlled trial. BMJ 336, 1416-1420.

3. Humphrey JH, Agoestina T, Wu L, et al. (1996) Impact of neonatal vitamin A supplementation on infant morbidity and mortality. J Pediatr 128, 489-496.

4. Rahmathullah L, Tielsch JM, Thulasiraj RD, et al. (2003) Impact of supplementing newborn infants with vitamin A on early infant mortality: community based randomised trial in southern India. BMJ 327, 254.

5. Benn CS, Rodrigues A, Yazdanbakhsh M, et al. (2009) The effect of high-dose vitamin A supplementation administered with BCG vaccine at birth may be modified by subsequent DTP vaccination. Vaccine 27, 2891-2898.

6. Benn CS, Fisker AB, Napirna BM, et al. (2010) Vitamin A supplementation and BCG vaccination at birth in low birthweight neonates: two by two factorial randomised controlled trial. BMJ 340, c1101.

7. Fisker AB, Lisse IM, Aaby P, et al. (2007) Effect of vitamin A supplementation with BCG vaccine at birth on vitamin A status at 6 wk and 4 mo of age. Am J Clin Nutr 86, 1032-1039.

8. da Silva ZJ, Oliveira I, Andersen A, et al. (2008) Changes in prevalence and incidence of HIV-1, HIV-2 and dual infections in urban areas of Bissau, Guinea-Bissau: is HIV-2 disappearing? AIDS 22, 1195-1202.

9. WHO (2010) WHO Recommendations for Routine Immunization - Summary Tables. http://www.who.int/entity/ immunization/policy/Immunization_routine_table2.pdf.

10. Diness BR, Fisker AB, Roth A, et al. (2007) Effect of highdose vitamin A supplementation on the immune response to Bacille Calmette-Guerin vaccine. Am J Clin Nutr 86, 1152-1159.

11. Erhardt JG, Estes JE, Pfeiffer CM, et al. (2004) Combined measurement of ferritin, soluble transferrin receptor, retinol binding protein, and C-reactive protein by an inexpensive, sensitive, and simple sandwich enzyme-linked immunosorbent assay technique. J Nutr 134, 3127-3132.

12. Agresti A (2002) Categorical Data Analysis, 2nd ed. New York: Wiley-Interscience.

13. Greene WH (2007) Econometric Analysis, 6th ed. Upper Saddle River, NJ: Pearson/Prentice Hall.

14. Uh HW, Hartgers FC, Yazdanbakhsh M, et al. (2008) Evaluation of regression methods when immunological measurements are constrained by detection limits. $B M C$ Immunol 9, 59

15. Barros AJ \& Hirakata VN (2003) Alternatives for logistic regression in cross-sectional studies: an empirical comparison of models that directly estimate the prevalence ratio. BMC Med Res Methodol 3, 21.

16. Wang X, Allen C \& Ballow M (2007) Retinoic acid enhances the production of IL-10 while reducing the synthesis of IL-12 and TNF-alpha from LPS-stimulated monocytes/ macrophages. J Clin Immunol 27, 193-200.

17. Mehta K, McQueen T, Tucker S, et al. (1994) Inhibition by all-trans-retinoic acid of tumor necrosis factor and nitric oxide production by peritoneal macrophages. J Leukoc Biol 55, 336-342.

18. DeCicco KL, Youngdahl JD \& Ross AC (2001) All-transretinoic acid and polyriboinosinic: polyribocytidylic acid in combination potentiate specific antibody production and cell-mediated immunity. Immunology 104, 341-348.

19. Cox SE, Arthur P, Kirkwood BR, et al. (2006) Vitamin A supplementation increases ratios of proinflammatory to antiinflammatory cytokine responses in pregnancy and lactation. Clin Exp Immunol 144, 392-400. 
20. Hoglen NC, Abril EA, Sauer JM, et al. (1997) Modulation of Kupffer cell and peripheral blood monocyte activity by in vivo treatment of rats with all-trans-retinol. Liver 17, 157-165.

21. Maynard CL, Hatton RD, Helms WS, et al. (2009) Contrasting roles for all-trans retinoic acid in TGF-beta-mediated induction of Foxp 3 and 1110 genes in developing regulatory T cells. $J$ Exp Med 206, 343-357.

22. Iwata M, Eshima Y \& Kagechika H (2003) Retinoic acids exert direct effects on T cells to suppress Th1 development and enhance Th2 development via retinoic acid receptors. Int Immunol 15, 1017-1025.

23. Damsgaard CT, Lauritzen L, Calder PC, et al. (2009) Wholeblood culture is a valid low-cost method to measure monocytic cytokines - a comparison of cytokine production in cultures of human whole-blood, mononuclear cells and monocytes. J Immunol Methods 340, 95-101.
24. Seguin-Devaux C, Hanriot D, Dailloux M, et al. (2005) Retinoic acid amplifies the host immune response to LPS through increased $\mathrm{T}$ lymphocytes number and LPS binding protein expression. Mol Cell Endocrinol 245, 67-76.

25. Coutsoudis A, Kiepiela P, Coovadia HM, et al. (1992) Vitamin A supplementation enhances specific IgG antibody levels and total lymphocyte numbers while improving morbidity in measles. Pediatr Infect Dis J 11, 203-209.

26. Ueki S, Mahemuti G, Oyamada H, et al. (2008) Retinoic acids are potent inhibitors of spontaneous human eosinophil apoptosis. J Immunol 181, 7689-7698.

27. Ahmad SM, Haskell MJ, Raqib R, et al. (2009) Markers of innate immune function are associated with vitamin a stores in men. J Nutr 139, 377-385.

28. Casimir GJ, Mulier S, Hanssens L, et al. (2010) Gender differences in inflammatory markers in children. Shock 33, $258-262$. 